\title{
Performance Comparison of Controllers with Fault-Dependent Control Allocation for UAVs
}

Nørgaard Sørensen, Mikkel Eske; Hansen, Søren; Breivik, Morten ; Blanke, Mogens

Published in:

Journal of Intelligent and Robotic Systems

Link to article, DOI:

$10.1007 / \mathrm{s} 10846-017-0494-9$

Publication date:

2017

Document Version

Peer reviewed version

Link back to DTU Orbit

Citation (APA):

Nørgaard Sørensen, M. E., Hansen, S., Breivik, M., \& Blanke, M. (2017). Performance Comparison of

Controllers with Fault-Dependent Control Allocation for UAVs. Journal of Intelligent and Robotic Systems, 87(1), 187-207. https://doi.org/10.1007/s10846-017-0494-9

\section{General rights}

Copyright and moral rights for the publications made accessible in the public portal are retained by the authors and/or other copyright owners and it is a condition of accessing publications that users recognise and abide by the legal requirements associated with these rights.

- Users may download and print one copy of any publication from the public portal for the purpose of private study or research.

- You may not further distribute the material or use it for any profit-making activity or commercial gain

- You may freely distribute the URL identifying the publication in the public portal 


\title{
Performance Comparison of Controllers with Fault-Dependent Control Allocation for UAVs
}

\author{
Mikkel Eske Nørgaard Sørensen ${ }^{1}$ • Søren Hansen ${ }^{2}$ • Morten Breivik ${ }^{1}$. \\ Mogens Blanke ${ }^{1,2}$
}

Received: date / Accepted: date

\begin{abstract}
This paper combines fault-dependent control allocation with three different control schemes to obtain fault tolerance in the longitudinal control of unmanned aerial vehicles. The paper shows that faultdependent control allocation is able to accommodate actuator faults that would otherwise be critical and it makes a performance assessment for the different control algorithms: an $\mathcal{L}_{1}$ adaptive backstepping controller; a robust sliding mode controller; and a standard PID controller. The actuator faults considered are the partial to total loss of the elevator, which is a critical component for the safe operation of unmanned aerial vehicles. During nominal operation, only the main actuator, namely the elevator, is active for pitch control. In the event of a partial or total loss of the elevator, faultdependent control allocation is used to redistribute control to available healthy actuators. Using simulations of a Cessna 182 aircraft model, controller performance and robustness are evaluated by metrics that assess control accuracy and energy use. System uncertainties are investigated over an envelope of pertinent variation, showing that sliding mode and $\mathcal{L}_{1}$ adaptive backstepping provide robustness, where PID control falls short. Additionally, a key finding is that the fault-dependent
\end{abstract}

${ }^{1}$ Centre for Autonomous Marine Operations and Systems,

Department of Engineering Cybernetics,

Norwegian University of Science and Technology,

NO-7491 Trondheim, Norway

E-mail:

mikkel.sorensen@itk.ntnu.no,

morten.breivik@ieee.org

${ }^{2}$ Automation and Control Group,

Department of Electrical Engineering,

Technical University of Denmark, Elektrovej B. 326,

DK-2800 Kgs. Lyngby, Denmark.

E-mail: sh@elektro.dtu.dk, mb@elektro.dtu.dk control allocation is instrumental when handling actuator faults.

Keywords Nonlinear longitudinal motion control . Fault-tolerant UAV control $\cdot \mathcal{L}_{1}$ adaptive backstepping control · Sliding mode control · Fault-dependent control allocation $\cdot$ Performance metrics

Acknowledgements This work was supported by the Research Council of Norway through the Centres of Excellence funding scheme, the AMOS project, grant number 223254.

\section{Introduction}

Critical safety issues must be considered when dealing with aircraft such as unmanned aerial vehicles (UAVs). In order to minimize risk of fault and failures, comprehensive checks are performed and meticulous maintenance is done regularly. Failures nevertheless occur, and actuator and control surfaces have particularly high criticality. Actuator redundancy can deal with some of the safety issues for UAVs, and fault-tolerant control (FTC) strategies can be employed to utilise such redundancy in the actuators.

Several different control methods have been applied to improve aircraft reliability. An overview of the recent development of FTC methods for aerial vehicles are given in [1], [2], [3] and [4]. Specific fault diagnosis approaches are treated in [5], [6], [7] and [8] related to control surfaces, and in [9] to the airspeed sensor system. Two control techniques, sliding mode control (SMC) [10], [11] and $\mathcal{L}_{1}$ adaptive control [12], are claimed to offer robust properties against matched uncertainties. The performance of SMC for attitude control of a fixedwing UAV is investigated in [13] where SMC is able to 
handle partial loss of a control surface. To make the system fault tolerant against the total loss of a control surface, a sliding mode observer is introduced in [14], making it possible to estimate a specific actuator fault. The authors of [15] use SMC on a linear aircraft model for FTC without the use of fault detection and isolation (FDI), which is referred to as passive FTC. The same authors implement SMC using control allocation in [16] and apply this method on the SIMONA research simulator in [17], showing that the controller is redistributed to the functioning actuator when a fault happens on the elevator. The $\mathcal{L}_{1}$ adaptive control technique is shown in [18] to be robust against faulty actuators, while the $\mathcal{L}_{1}$ adaptive backstepping control (L1-AB) technique is used in [19] as the pitch autopilot for an agile missile.

Control allocation (CA) is based on separating the control law from the signal distribution task, which gives CA the possibility to be combined with many different control laws. This is done in [20] by designing a controller to provide a "virtual control" which is mapped to the actual control signals sent to the actuators. The CA approach can manage the redundancy of an over-actuated system [21]. The combination of L1$\mathrm{AB}$ and $\mathrm{CA}$ is explored in [22] to control an F16 in a fault-free case. In [23], a fault-dependent control allocation scheme was developed and combined with L1-AB. In [1], SMC and CA is combined to analyse the performance for FTC applications. A further improvement is proposed in [24] using an integral sliding mode (ISM), which combines a controller that handles uncertainties of a system with the sliding mode control. If the system is subjected to external bounded disturbances, the ISM will compensate using sliding mode control while the original controller handles the unperturbed system [25]. In [26], an FTC structure which handles control surface failure is introduced by a combined use of generalized dynamic inversion control and ISM control. An over-actuated aircraft can easily maintain the required forces and moments even though a fault has occurred by applying the CA approaches suggested in [1] and [25]. However, many small UAVs are not over-actuated and hence using the CA from [1] is not possible.

This paper is based on the work in [23]. Here, we suggest a new control allocation approach to handle the non-over-actuated control surface configuration usually found on smaller aircraft. Balancing obtainable forces and moments, the $\mathrm{CA}$ is shown to help achieve a necessary flight envelope in case of faults, and also being able to prioritize such that stabilisability is preserved. The fault-hiding property of the CA is then utilised by stabilising control to obtain a total fault-tolerant con- trol system. The achievable performance is compared for three controller designs: A conventional PID, a robust controller in the form of sliding mode and an adaptive controller in the form of $\mathcal{L}_{1}$ adaptive backstepping. The PID is employed being a widely used baseline design in industry. Simulation results are made using a high-fidelity aircraft model [27] of a Cessna 182 with non-redundant actuation: an elevator to control pitch; a rudder to mainly control yaw; a pair of ailerons to control roll; and a throttle to control forward thrust. It is shown that the fault-dependent control allocation makes it possible for all the considered controllers to achieve excellent tracking performance even though a fault is occurring, and that the controllers have the ability to compensate for internal uncertainties. Additionally, it is shown that an adaptive controller in the form of $\mathcal{L}_{1}$ adaptive backstepping and a robust controller in the form of sliding mode perform better than the PID controller.

The structure of the paper is as follows: A mathematical model, assumptions and fault modelling are presented in Section 2; Section 3 deals with control allocation; Section 4 presents the controllers developed for the fixed-wing UAV; Section 5 includes definition of metrics, simulation results and performance evaluation obtained from the combination of the control laws and the control allocation; while Section 6 concludes the paper.

\section{Aircraft Dynamics}

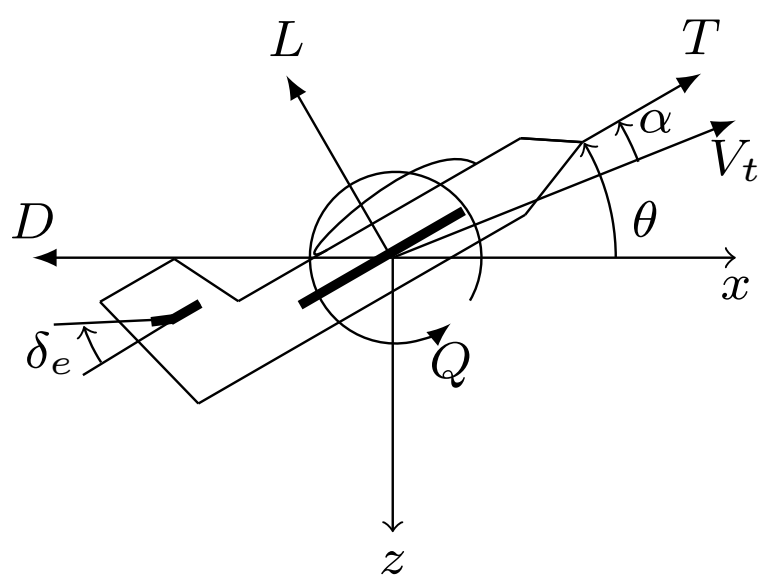

Fig. 1: Schematic of the longitudinal motion of an aircraft

Longitudinal aircraft motion is considered, where the state vector $\mathbf{x}_{\text {lon }} \triangleq\left[\theta, Q, \alpha, V_{t}\right]^{\top}$ is defined, with 
the components pitch angle $\theta[\mathrm{rad}]$, pitch rate $Q[\mathrm{rad} / \mathrm{s}]$, angle of attack $\alpha[\mathrm{rad}]$ and true airspeed $V_{t}[\mathrm{~m} / \mathrm{s}]$, see Fig. 1. The dynamics of the longitudinal aircraft model can be stated as [28]:

$$
\begin{aligned}
\dot{\theta}= & Q \\
I_{y} \dot{Q}= & \bar{m}\left(Q, \alpha, \dot{\alpha}, V_{t}, \delta_{e}\right) \\
= & \bar{q} \bar{S} \bar{c}\left(c_{m 0}+c_{m \alpha}^{*} \alpha+c_{m \delta e} \delta_{e}\right. \\
& \left.+\frac{\bar{c}}{2 V_{t}}\left(c_{m \dot{\alpha}}^{*} \dot{\alpha}+c_{m Q}^{*} Q\right)\right) \\
m V_{t} \dot{\alpha}= & m g \cos (\theta-\alpha)-T \sin (\alpha)-L+m V_{t} Q \\
m \dot{V}_{t}= & T \cos (\alpha)-D-m g \sin (\theta-\alpha),
\end{aligned}
$$

where $m>0[\mathrm{~kg}]$ is the mass of the aircraft, $g=9.81$ $\left[\mathrm{m} / \mathrm{s}^{2}\right]$ the gravity constant, $I_{y}$ the y-moment of inertia, $\bar{q}=\frac{1}{2} \rho V_{t}^{2}$ the dynamic pressure, $\bar{S}$ wing area, $\bar{c}$ the mean aerodynamic chord of the wing, $\rho$ air density, $\delta_{e}$ deflection angle of the elevator and $T$ engine thrust, which is modelled as

$T=\frac{\eta \delta_{t}}{V_{t}}$

where $\eta$ is the propeller efficiency [\%] and $\delta_{t}$ is input power $[\mathrm{W}]$. Furthermore, the relations between the drag $D$, lift $L$, side force $Y$, roll moment $\bar{l}$, pitch moment $\bar{m}$, yaw moment $\bar{n}$ and the deflection of the control surfaces are [28]:

$$
\begin{aligned}
D & =\bar{q} \bar{S}\left[c_{D}\left(\mathbf{x}_{l o n}, \delta_{e}\right)+\Delta_{D}\left(\mathbf{x}_{l o n}, \delta_{e}\right)\right] \\
L & =\bar{q} \bar{S}\left[c_{L}\left(\mathbf{x}_{l o n}, \delta_{e}\right)+\Delta_{L}\left(\mathbf{x}_{l o n}, \delta_{e}\right)\right] \\
Y & =\bar{q} \bar{S}\left[c_{Y}\left(\mathbf{x}_{l a t}, \delta_{a}, \delta_{r}\right)+\Delta_{Y}\left(\mathbf{x}_{l a t}, \delta_{a}, \delta_{r}\right)\right] \\
\bar{l} & =\bar{q} \bar{S} b\left[c_{l}\left(\mathbf{x}_{l a t}, \delta_{a}, \delta_{r}\right)+\Delta_{l}\left(\mathbf{x}_{l a t}, \delta_{a}, \delta_{r}\right)\right] \\
\bar{m} & =\bar{q} \bar{S} \bar{c}\left[c_{m}\left(\mathbf{x}_{l o n}, \delta_{e}\right)+\Delta_{m}\left(\mathbf{x}_{l o n}, \delta_{e}\right)\right] \\
\bar{n} & =\bar{q} \bar{S} b\left[c_{n}\left(\mathbf{x}_{l a t}, \delta_{a}, \delta_{r}\right)+\Delta_{n}\left(\mathbf{x}_{l a t}, \delta_{a}, \delta_{r}\right)\right],
\end{aligned}
$$

where $\mathbf{x}_{l a t} \triangleq[\phi, \psi, P, R, \beta]^{\top}$ represents the lateral aircraft states, $b$ the wing span, $\delta_{a}$ the deflection angle of the ailerons and $\delta_{r}$ the deflection angle of the rudder. The $\Delta_{i}(\mathbf{x}, \boldsymbol{\delta})$ terms, where the index $i$ refers to forces and moments of (6)-(11), are unmodelled dynamics caused by uncertainty of the aerodynamic coefficients. It is assumed that $\Delta_{i}(\mathbf{x}, \boldsymbol{\delta})$ are unknown but bounded, as

$\left\|\Delta_{i}(\mathbf{x}, \boldsymbol{\delta})\right\| \leq v_{i}(\mathbf{x}, \boldsymbol{\delta})$,

where $v_{i}(\mathbf{x}, \boldsymbol{\delta})>0$ is a known function.

\subsection{Assumptions}

The control objective is to track a reference signal of the pitch angle $\theta_{d}$, see Section 4 . In order to design a controller which fulfils this control objective, it is assumed that the true airspeed can be controlled separately and therefore can be neglected from the pitch controller design.

The angle of attack and true airspeed need to meet the conditions:

$$
\begin{aligned}
|\alpha| & \leq \alpha_{\max } \\
|\dot{\alpha}| & \leq \dot{\alpha}_{\max } \\
V_{t, \min } \leq V_{t} & \leq V_{t, \max }
\end{aligned}
$$

The stall condition and the fear of structural damage to the wings are the reasons for these assumptions. It is assumed that uncertainties only exist in the coefficients of the pitch moment $\bar{m}$ and that the aerodynamic coefficients $c_{m 0}$ and $c_{m \delta e}$ are known. In [29] and [30], it is shown through system identification that these coefficients are almost perfectly identified, which gives the justification for this assumption about these coefficients. In [31], uncertainty in the control signal and external disturbances are also considered.

For the rest of the aerodynamic coefficients, the relationship between the real and assumed coefficients is parametrised as

$$
\begin{aligned}
c_{m \alpha}^{*} & =\sigma_{\alpha} c_{m \alpha} \\
c_{m \dot{\alpha}}^{*} & =\sigma_{\dot{\alpha}} c_{m \dot{\alpha}} \\
c_{m Q}^{*} & =\sigma_{Q} c_{m Q},
\end{aligned}
$$

where $c_{m i}^{*}$ represents the true coefficients, $\sigma_{\alpha} \in \mathbb{R}^{+}$is the uncertainty associated with the coefficient of pitch moment with respect to the angle of attack, $\sigma_{\dot{\alpha}} \in \mathbb{R}^{+}$is the uncertainty associated with the coefficient of pitch moment with respect to the derivative of the angle of attack, and $\sigma_{Q} \in \mathbb{R}^{+}$is the uncertainty associated with the coefficient of pitch moment with respect to the pitch rate. Additionally, it is assumed that $\dot{\sigma}_{\alpha}=\dot{\sigma}_{\dot{\alpha}}=\dot{\sigma}_{Q}=$ 0 , i.e. the uncertainties are constant or slowly varying relative to the aircraft dynamics.

Using these assumptions, the longitudinal motion in (1)-(4) becomes

$$
\begin{aligned}
\dot{\theta}= & Q \\
I_{y} \dot{Q}= & \bar{q} \bar{S} \bar{c}\left(c_{m 0}+\sigma_{\alpha} c_{m \alpha} \alpha+c_{m \delta e} \delta_{e}\right. \\
& \left.+\frac{\bar{c}}{2 V_{t}}\left(\sigma_{\dot{\alpha}} c_{m \dot{\alpha}} \dot{\alpha}+\sigma_{Q} c_{m Q} Q\right)\right) .
\end{aligned}
$$




\subsection{Possible Faults}

The following fault models are based on [1] and [15]. In this paper, it is assumed that only the elevator can be faulty. Elevator faults can be categorised as:

(a) Partial faults, which are commonly occurring in small aircraft. A partial actuator fault can be modelled as $\boldsymbol{\delta}^{e}(t)=\mathbf{W}(t) \boldsymbol{\delta}(t)$

where $\boldsymbol{\delta}^{e} \in \mathbb{R}^{3}$ is the effective control vector, $\boldsymbol{\delta} \in$ $\mathbb{R}^{3}$ is the control vector and $\mathbf{W}(t)$ represents the effectiveness of the actuators. The matrix $\mathbf{W}(t) \in$ $\mathbb{R}^{3 \times 3}$ is defined as

$\mathbf{W}(t) \triangleq \operatorname{diag}\left(w_{1}(t), w_{2}(t), w_{3}(t)\right)=\mathbf{I}-\mathbf{K}(t)$,

where $\mathbf{I} \in \mathbb{R}^{3 \times 3}$ is the identity matrix and $\mathbf{K}(t) \in$ $\mathbb{R}^{3 \times 3}$ is the multiplicative fault matrix, which is defined for the aircraft as

$\mathbf{K}(t) \triangleq \operatorname{diag}\left(k_{1}(t), k_{2}(t), k_{3}(t)\right)$

with $k_{i}(t) \in[0,1)$, which is associated with the elevator, ailerons and rudder control surfaces. Here, $k_{i}=0$ means that the $i$ th control surface is in a fault-free condition, while $k_{i}=1$ indicates that a total loss of effectiveness on the $i$ th control surface has occurred.

(b) Total faults, which include

- Stuck-in-place, which is defined as an actuator stuck at a fixed position being immovable

- Hard-over, which is a special case of a stuckin-place fault, where an actuator is stuck at an extreme position being immovable

- Loss of control surface.

A total fault is modelled as

$\delta_{i}^{e}(t)=k_{i} \delta_{i, f}$,

where $\delta_{i, f}$ is the control input which the $i$ th control surface receives if it has a total fault. If the fault that has occurred is a stuck-in-place actuator fault then $\delta_{i, f}=c$ with $c \in\left[\delta_{i, \min }, \delta_{i, \max }\right]$ and $\dot{c}=0$. A hard-over fault is a special case of a stuck-in-place fault where $\delta_{i, f}=\delta_{i, \min }$ or $\delta_{i, f}=\delta_{i, \max }$. A total loss of effectiveness on the $i$ th control surface could also occur if the control surface is detached from the plane, i.e. $\delta_{i, f}=0$.

It is assumed in this paper that we have a fault detection and isolation scheme which is able to estimate the faults. For a real-world scenario, $\mathbf{W}(t)$ in $(22)$ can be obtained by a separate fault identification scheme, see e.g. [1], [4], [9] and [32].

\section{Fault-dependent Control Allocation}

The role of the Control allocation (CA) is the following: Given commanded forces and moments from the controllers, calculate deflection of the control surfaces such that the commands are fulfilled. "A control allocation algorithm's primary objective is to compute a control input that ensures that the virtual control command is produced jointly by the effectors at all time" [20]. A general schematic of the proposed fault-dependent control allocation scheme is displayed in Fig. 2.

Three quantities are particularly important to derive the deflection angles on control surfaces: lift force, roll moment and yaw moment. The lift force ensures the aircraft remains airborne, while the roll and yaw moment are needed to stabilize the aircraft. We note that the control allocation does not take into account that some uncertainty exist in the pitch moment. For notational simplicity, time $t$ is omitted in the following. Omitting the uncertainties $\Delta_{i}(\mathbf{x}, \boldsymbol{\delta})$, the lift force, roll moment and yaw moment in (7), (9) and (11) are

$$
\begin{aligned}
L & =\bar{q} \bar{S}\left(c_{L}\left(\mathbf{x}_{\text {lon }}\right)+\frac{\partial c_{L}}{\partial \delta_{e}} \delta_{e}\right) \\
\bar{l} & =\bar{q} \bar{S} b\left(c_{l}\left(\mathbf{x}_{l a t}\right)+\frac{\partial c_{l}}{\partial \delta_{a}} \delta_{a}+\frac{\partial c_{l}}{\partial \delta_{r}} \delta_{r}\right) \\
\bar{n} & =\bar{q} \bar{S} b\left(c_{n}\left(\mathbf{x}_{l a t}\right)+\frac{\partial c_{n}}{\partial \delta_{a}} \delta_{a}+\frac{\partial c_{n}}{\partial \delta_{r}} \delta_{r}\right) .
\end{aligned}
$$

In the fault-free case, the deflection angles on the control surfaces are

$$
\begin{aligned}
\delta_{e, n o m} & =\frac{1}{\bar{q} \bar{S} \frac{\partial c_{L}}{\partial \delta_{e}}}\left(L-\bar{q} \bar{S} c_{L}\left(\mathbf{x}_{l o n}\right)\right) \\
\delta_{a, n o m} & =\frac{1}{\bar{q} \bar{S} b \frac{\partial c_{l}}{\partial \delta_{a}}}\left(\bar{l}-\bar{q} \bar{S} b\left(c_{l}\left(\mathbf{x}_{l a t}\right)+\frac{\partial c_{l}}{\partial \delta_{r}} \delta_{r}\right)\right)
\end{aligned}
$$

$$
\begin{aligned}
\delta_{r, n o m}= & \frac{1}{\bar{q} \bar{S} b\left(\frac{\partial c_{l}}{\partial \delta_{a}} \frac{\partial c_{n}}{\partial \delta_{r}}-\frac{\partial c_{l}}{\partial \delta_{r}} \frac{\partial c_{n}}{\partial \delta_{a}}\right)}\left(\frac { \partial c _ { n } } { \partial \delta _ { a } } \left(\bar{q} \bar{S} b c_{l}\left(\mathbf{x}_{l a t}\right)\right.\right. \\
& \left.-\bar{l})+\frac{\partial c_{l}}{\partial \delta_{a}}\left(\bar{n}-\bar{q} \bar{S} b c_{n}\left(\mathbf{x}_{l a t}\right)\right)\right)
\end{aligned}
$$

where it is assumed that the aerodynamic coefficients $\frac{\partial c_{L}}{\partial \delta_{e}}, \frac{\partial c_{l}}{\partial \delta_{a}}, \frac{\partial c_{l}}{\partial \delta_{r}}, \frac{\partial c_{n}}{\partial \delta_{a}}$ and $\frac{\partial c_{n}}{\partial \delta_{r}}$ are constant and nonzero around a stationary condition. This assumption is based on results from wind tunnel tests [33].

Saturation of deflection angle is

$\delta_{i, \min } \leq \delta_{i} \leq \delta_{i, \max }$,

where the index $i$ refers to elevator, ailerons or rudder. 


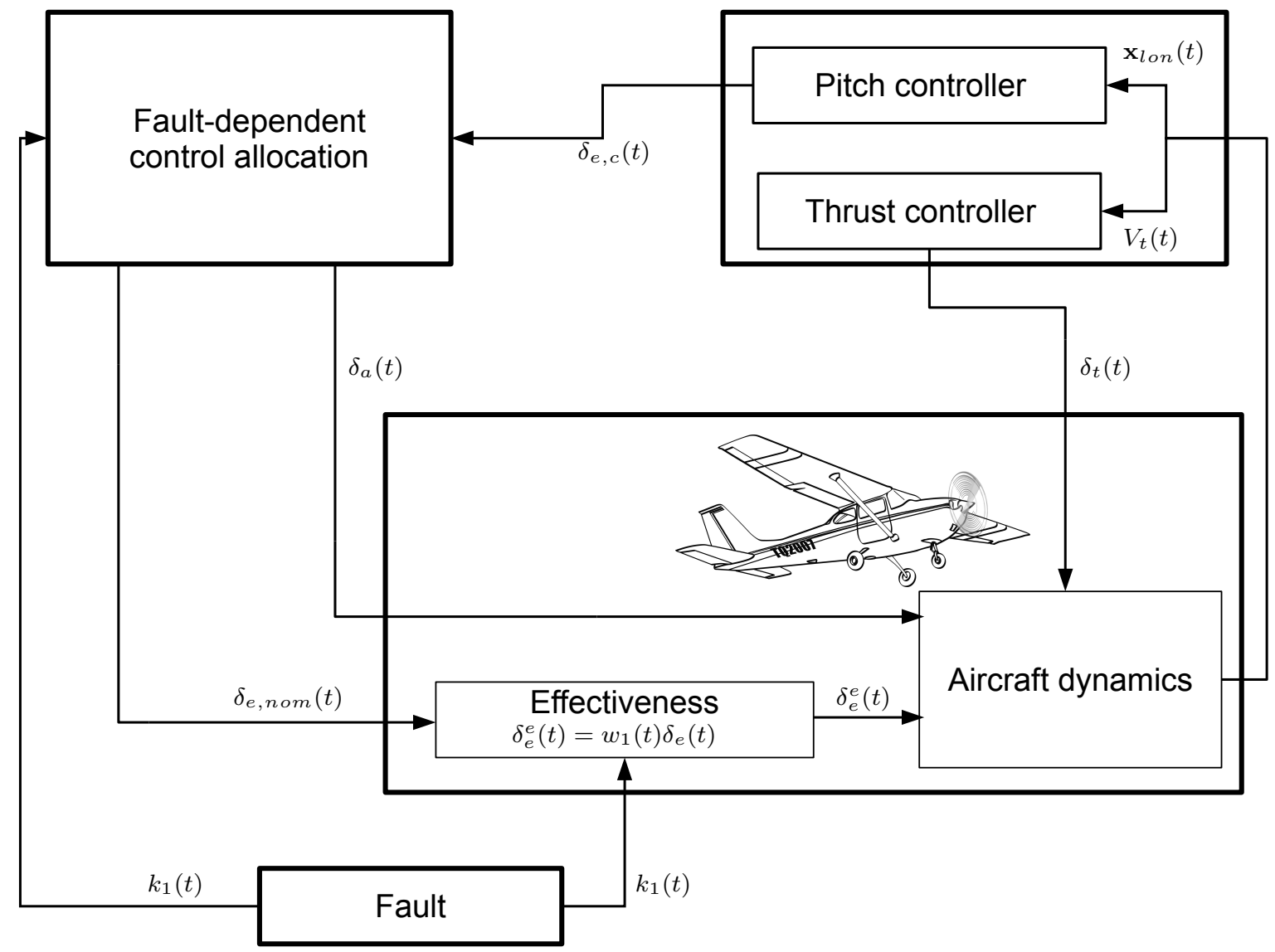

Fig. 2: Schematic of the fault-dependent control allocation implementation

\subsection{Handling of Faults}

In the following example, we consider a scenario where the elevator is faulty. However, this procedure can be incorporated into a general case. To overcome a partial or total elevator fault, the ailerons are subsequently reconfigured such that they work as a second set of elevators. The deflection angle for the ailerons $\delta_{a}$ is then recalculated for the drag force, lift force and pitch moment. In this paper, it is desired to control the pitch moment, which also affects the other inputs in the longitudinal system. The deflection angle for the ailerons $\delta_{a}$ is a function of effectiveness on the elevator, where the goal is to maintain the pitch moment $\bar{m}$

$\delta_{a}=\frac{1}{\bar{q} \bar{S} \bar{c} \frac{\partial c_{m}}{\partial \delta_{a}}}\left(\bar{m}-\bar{q} \bar{S} \bar{c}\left(c_{m}\left(\mathbf{x}_{l o n}\right)+\frac{\partial c_{m}}{\partial \delta_{e}} w_{1} \delta_{e}\right)\right)$.

The CA approach for the elevator is evaluated in Fig. 3 over the range $\left[\delta_{e, \min }, \delta_{e, \max }\right]$ for the fault-free case and with a partial loss on the elevator. The input for the evaluation is the demanded deflection on the elevator $\delta_{e, n o m}$, which has a limitation of [-22:18] degrees, and the output is the pitch moment which the fault-dependent control allocation using the aileron redistribution manages to obtain. Fig. 3 also shows the difference between the fault-free and the faulty cases and from which set point it is possible to maintain the same pitch moment by compensating the loss of the elevator with the ailerons. The span where the error is zero will decrease with the loss of effectiveness of the elevator.

A method to improve the allocation span is

$$
\begin{aligned}
\delta_{e} & =\frac{\delta_{e, n o m}}{w_{1}+\varrho} \\
\delta_{a} & =\frac{1}{\bar{q} \bar{S} \bar{c} \frac{\partial c_{m}}{\partial \delta_{a}}}\left(\bar{m}-\bar{q} \bar{S} \bar{c}\left(c_{m}\left(\mathbf{x}_{l o n}\right)+\frac{\partial c_{m}}{\partial \delta_{e}} \delta_{e} w_{1}\right)\right),
\end{aligned}
$$

where $\varrho>0$ is a small positive constant. However, this is only possible if the elevator is not saturated and only if it is a partial fault. The result of the approach in (33)-(34) gives a small improvement in the span where the pitch moment of the fault-free and faulty cases are 
The pitch moment for the fault-free case

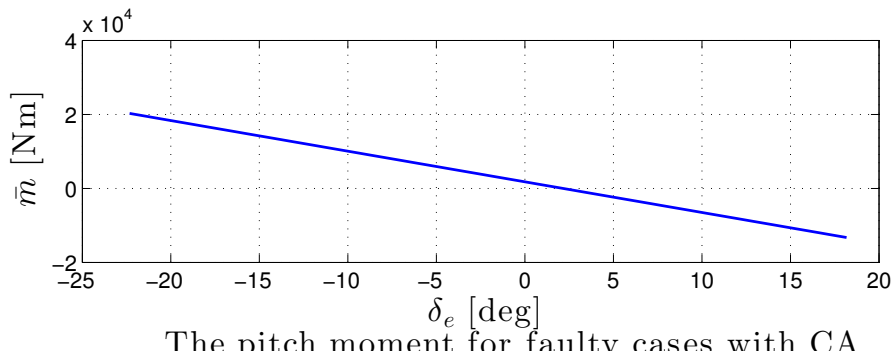

The pitch moment for faulty cases with CA

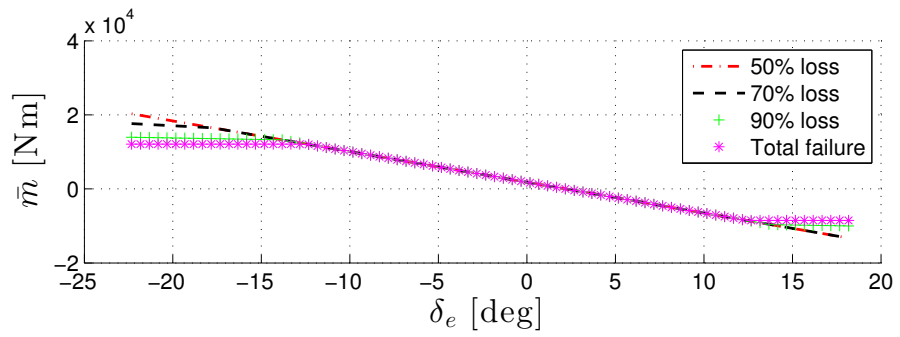

Error between fault-free and faulty cases with CA



Fig. 3: Plots of the fault-free, faulty and difference between the fault-free and faulty cases with fault-dependent control allocation for the pitch moment

the same.

In order to compensate for model uncertainties, a robust controller in the form of sliding mode and an adaptive controller in the form of $\mathcal{L}_{1}$ adaptive backstepping, which are known to handle unknown system parameters, are implemented. Additionally, a PID controller is implemented as a baseline.

\section{Controller Design}

This section presents procedures for a conventional PID controller, a robust controller in the form of sliding mode and an adaptive controller in the form of $\mathcal{L}_{1}$ adaptive backstepping for the longitudinal dynamics. The controller receives the pitch angle $\theta$ and pitch rate $Q$ and gives a commanded control input as the deflection angle on the elevator $\delta_{e, c}$, which is converted into demanded force and moment in the control allo- cation block. The control objective is to make $\mid \theta(t)-$ $\theta_{d}(t) \mid \rightarrow 0$, where the desired pitch angle $\theta_{d}(t)$ is $\mathcal{C}^{2}$ and bounded. This reference signal is typically defined by a human or generated by a guidance system. For notational simplicity, time $t$ is omitted in the following.

\subsection{PID Control}

The control law for a PID controller can be chosen as

$$
\delta_{e, c}=K_{p, \theta} \frac{\tau_{i} s+1}{\tau_{i} s} \frac{\tau_{d} s+1}{a \tau_{d} s+1} e_{p},
$$

where

$e_{p} \triangleq \theta_{d}-\theta$

By making an input-output linearisation of (19)-(20) and since it is known from [33] that $c_{m}(\cdot)<0$, the transfer function has a negative numerator, which changes 
the control law to

$\delta_{e, c}=-K_{p, \theta} \frac{\tau_{i} s+1}{\tau_{i} s} \frac{\tau_{d} s+1}{a \tau_{d} s+1} e_{p}$,

We have additionally introduced an anti-windup method to the PID controller since the elevator can saturate.

\subsection{Sliding Mode Control (SMC)}

The SMC design is divided into two stages: The first concerns the design of the sliding surface, while the second stage is designing the control law where the sliding mode is obtained. For the design of the control law, the assumed model parameters in Section 2.1 are used.

\subsubsection{Sliding Surface Design}

First, a sliding surface is defined by $\mathcal{S} \triangleq\{\mathbf{e}: s=0\}$, where $\mathbf{e}$ is vector of tracking errors and $s$ is the switching function. The design of this sliding surface is formulated in [10] and [34], while this paper will use an approach inspired by [34].

The error signals are

$e_{1} \triangleq \theta-\theta_{d}$

$e_{2} \triangleq \dot{e_{1}}=\dot{\theta}-\dot{\theta}_{d}=Q-Q_{d}$

and their derivatives are

$\dot{e}_{1}=e_{2}$

$\dot{e}_{2}=\dot{Q}-\dot{Q}_{d}$.

Let the sliding surface be

$s=e_{2}+A_{1} e_{1}=0, \quad A_{1}>0$.

On this surface, the motion is governed by

$\dot{e}_{1}=-A_{1} e_{1}$.

\subsubsection{Control Law Design}

The derivative of the switching function $s$ can be expressed as

$$
\begin{aligned}
\dot{s}= & \dot{e_{2}}+A_{1} \dot{e_{1}} \\
= & \frac{\bar{q} \bar{S} \bar{c}}{I_{y}}\left(c_{m 0}+c_{m \alpha} \alpha+c_{m \delta e} \delta_{e}\right. \\
& \left.+\frac{\bar{c}}{2 V_{t}}\left(c_{m \dot{\alpha}} \dot{\alpha}+c_{m Q}\left(s+Q_{d}-A_{1} e_{1}\right)\right)\right) \\
& -\dot{Q}_{d}+A_{1} e_{2} .
\end{aligned}
$$

For SMC, a sign function is ideally used to force $s \rightarrow 0$ in finite time. However, instead of the sign function, a saturation function has been chosen to minimise chattering. To ensure stability, the use of feedback control needs to turn (44) into a negative definite function, which is shown by (54). The input $\delta_{e}$ is chosen to be

$$
\delta_{e, c}=\frac{-1}{c_{m \delta e}}\left(c_{m 0}+\frac{I_{y}}{\bar{q} \bar{S} \bar{c}}\left(A_{1} e_{2}-\dot{Q}_{d}\right)-v\right),
$$

where $v$ is an additional control signal. Inserting $\delta_{e}$ from (45) into (44), the switching function is rewritten as

$\dot{s}=\frac{\bar{q} \bar{S} \bar{c}}{I_{y}}(v+\zeta)$,

where

$\zeta=c_{m \alpha} \alpha+\frac{\bar{c}}{2 V_{t}}\left(c_{m \dot{\alpha}} \dot{\alpha}+c_{m Q}\left(s+Q_{d}-A_{1} e_{1}\right)\right)$

such that

$$
\begin{aligned}
|\zeta| & \leq\left|c_{m \alpha}\right| \alpha_{\max }+\frac{\bar{c}}{2 V_{t, \min }}\left(\left|c_{\operatorname{mi}}\right| \dot{\alpha}_{\max }\right. \\
& \left.+\left|c_{m Q}\right|\left|s+Q_{d}-A_{1} e_{1}\right|\right) .
\end{aligned}
$$

Then taking

$v=-\beta \operatorname{sat}\left(\frac{s}{\varepsilon}\right), \varepsilon>0$

and

$$
\begin{aligned}
\beta & \geq\left|c_{\operatorname{m\alpha }}\right| \alpha_{\max }+\frac{\bar{c}}{2 V_{t_{\min }}}\left(\left|c_{\operatorname{mi}}\right| \dot{\alpha}_{\max }\right. \\
& \left.+\left|c_{m Q}\right|\left|s+Q_{d}-A_{1} e_{1}\right|\right)+\beta_{0},
\end{aligned}
$$

where $\beta_{0}>0$.

The derived control law is assessed by using a positive definite control Lyapunov function (CLF)

$V_{S M C, 1}=\frac{1}{2} s^{2}$.

Taking the derivative yields

$$
\begin{aligned}
\dot{V}_{S M C, 1}= & s \dot{s}=s \frac{\bar{q} \bar{S} \bar{c}}{I_{y}}\left(c_{m \alpha} \alpha+\frac{\bar{c}}{2 V_{t}}\left(c_{m \dot{\alpha}} \dot{\alpha}\right.\right. \\
& \left.\left.+c_{m Q}\left(s+Q_{d}-A_{1} e_{1}\right)\right)-\beta\right) .
\end{aligned}
$$

By inserting $\beta$, it can be said that for $|s| \geq \varepsilon$,

$s \dot{s} \leq-\beta_{0}|s| \frac{\bar{q} \bar{S} \bar{c}}{I_{y}}$,

which implies that the trajectories reach the boundary layer $\{|s| \leq \varepsilon\}$ in finite time. Inside the boundary layer

$\dot{e}_{1}=-A_{1} e_{1}+s$, 
and choosing the CLF $V_{S M C, 2}=\frac{1}{2} e_{1}^{2}$ and where $|s| \leq \varepsilon$, the derivative is

$\dot{V}_{S M C, 2}=e_{1} \dot{e_{1}}=-A_{1} e_{1}^{2}+e_{1} s \leq-A_{1} e_{1}^{2}+\left|e_{1}\right| \varepsilon$.

By taking $A_{1}=2$

$e_{1} \dot{e_{1}} \leq-2 e_{1}^{2}+\left|e_{1}\right| \varepsilon \leq-e_{1}^{2}, \forall\left|e_{1}\right| \geq \varepsilon$.

By choosing this method it is not possible to stabilise the origin but it achieves ultimate boundedness with an ultimate bound which can be reduced by decreasing $\varepsilon$. From this knowledge, one should be aware of a small stationary control error. Finally, the commanded control signal becomes

$$
\begin{aligned}
\delta_{e, c}= & \frac{-1}{c_{m \delta e}}\left(c_{m 0}+\frac{I_{y}}{\bar{q} \bar{S} \bar{c}}\left(2 e_{2}-\dot{Q}_{d}\right)\right. \\
& \left.+\beta \operatorname{sat}\left(\frac{s}{\varepsilon}\right)\right) .
\end{aligned}
$$

\section{$4.3 \mathcal{L}_{1}$ Adaptive Backstepping Control (L1-AB)}

The design of the $\mathcal{L}_{1}$ adaptive backstepping controller can also be performed in two steps: The first stage concerns the design of the adaptation laws, while the second stage focus on the control law. The design is inspired by the approach in [19].

\subsubsection{State Predictor}

The prediction errors $\tilde{\theta}$ and $\tilde{Q}$ are defined as

$$
\begin{gathered}
\tilde{\theta} \triangleq \hat{\theta}-\theta \\
\tilde{Q} \triangleq \hat{Q}-Q,
\end{gathered}
$$

where $\hat{\theta}, \hat{Q}, \theta$ and $Q$ represent the estimated pitch angle, estimated pitch rate, real pitch angle and real pitch rate, respectively. The desired prediction error dynamics are chosen to be

$$
\begin{gathered}
\dot{\tilde{\theta}}_{i d e a l}=-L_{1} \tilde{\theta} \\
\dot{\tilde{Q}}_{\text {ideal }}=-L_{2} \tilde{Q},
\end{gathered}
$$

where the convergence rate is defined through the positive gains $L_{1}>0$ and $L_{2}>0$, to ensure that their origins are exponentially stable. From the latter, the state prediction dynamics are given as

$$
\begin{aligned}
\dot{\hat{\theta}}= & -L_{1} \tilde{\theta}+Q \\
\dot{\hat{Q}}= & -L_{2} \tilde{Q}+\frac{\bar{q} \bar{S} \bar{c}}{I_{y}}\left(c_{m 0}+\hat{\sigma}_{\alpha} c_{m \alpha} \alpha+c_{m \delta e} \delta_{e}\right. \\
& \left.+\frac{\bar{c}}{2 V_{t}}\left(\hat{\sigma}_{\dot{\alpha}} c_{m \dot{\alpha}} \dot{\alpha}+\hat{\sigma}_{Q} c_{m Q} Q\right)\right),
\end{aligned}
$$

where $\hat{\sigma}_{\alpha}, \hat{\sigma}_{\dot{\alpha}}$ and $\hat{\sigma}_{Q}$ are the estimates of the aerodynamic parameter uncertainties. The design of adaptation laws for the uncertainties is based on Lyapunov stability analysis. Substituting (19), (20), (63) and (64) into (59) and (60), the prediction error dynamics become

$$
\begin{aligned}
\dot{\tilde{\theta}}= & -L_{1} \tilde{\theta} \\
\dot{\tilde{Q}}=- & L_{2} \tilde{Q}+\frac{\bar{q} \bar{S} \bar{c}}{I_{y}}\left(\tilde{\sigma}_{\alpha} c_{m \alpha} \alpha+\frac{\bar{c}}{2 V_{t}}\left(\tilde{\sigma}_{\dot{\alpha}} c_{m \dot{\alpha}} \dot{\alpha}\right.\right. \\
& \left.\left.+\tilde{\sigma}_{Q} c_{m Q} Q\right)\right) .
\end{aligned}
$$

Let's consider the positive definite CLF

$$
V_{\text {pred }}=\frac{1}{2}\left(\frac{1}{\gamma_{\alpha}} \tilde{\sigma}_{\alpha}^{2}+\frac{1}{\gamma_{\dot{\alpha}}} \tilde{\sigma}_{\dot{\alpha}}^{2}+\frac{1}{\gamma_{Q}} \tilde{\sigma}_{Q}^{2}\right)+\frac{1}{2} \tilde{\theta}^{2}+\frac{1}{2} \tilde{Q}^{2} .
$$

Taking the time derivative of (67) yields

$$
\begin{aligned}
\dot{V}_{\text {pred }}= & \frac{1}{\gamma_{\alpha}} \tilde{\sigma}_{\alpha} \dot{\tilde{\sigma}}_{\alpha}+\frac{1}{\gamma_{\dot{\alpha}}} \tilde{\sigma}_{\dot{\alpha}} \dot{\tilde{\sigma}}_{\dot{\alpha}}+\frac{1}{\gamma_{Q}} \tilde{\sigma}_{Q} \dot{\tilde{\sigma}}_{Q}-L_{1} \tilde{\theta}^{2}-L_{2} \tilde{Q}^{2} \\
& +\tilde{Q}\left(\frac { \overline { q } \overline { S } \overline { c } } { I _ { y } } \left(\tilde{\sigma}_{\alpha} c_{m \alpha} \alpha+\frac{\bar{c}}{2 V_{t}}\left(\tilde{\sigma}_{\dot{\alpha}} c_{m \dot{\alpha}} \dot{\alpha}\right.\right.\right. \\
& \left.\left.\left.+\tilde{\sigma}_{Q} c_{m Q} Q\right)\right)\right) .
\end{aligned}
$$

Since it is assumed that $\dot{\sigma}_{\alpha}=\dot{\sigma}_{\dot{\alpha}}=\dot{\sigma}_{Q}=0,(68)$ can be rewritten as

$$
\begin{aligned}
\dot{V}_{\text {pred }}= & -L_{1} \tilde{\theta}^{2}-L_{2} \tilde{Q}^{2}+\hat{\sigma}_{\alpha}\left(\frac{1}{\gamma_{\alpha}} \dot{\hat{\sigma}}_{\alpha}+\tilde{Q} \frac{\bar{q} \bar{S} \bar{c}}{I_{y}} c_{m \alpha} \alpha\right) \\
& +\hat{\sigma}_{\dot{\alpha}}\left(\frac{1}{\gamma_{\dot{\alpha}}} \dot{\hat{\sigma}}_{\dot{\alpha}}+\tilde{Q} \frac{\bar{q} \bar{S} \bar{c}}{I_{y}} \frac{\bar{c}}{2 V_{t}} c_{m \dot{\alpha}} \dot{\alpha}\right) \\
& +\hat{\sigma}_{Q}\left(\frac{1}{\gamma_{Q}} \dot{\hat{\sigma}}_{Q}+\tilde{Q} \frac{\bar{q} \bar{S} \bar{c}}{I_{y}} \frac{\bar{c}}{2 V_{t}} c_{m Q} Q\right) .
\end{aligned}
$$

To eliminate the uncertainty terms $\hat{\sigma}_{\alpha}, \hat{\sigma}_{\dot{\alpha}}$ and $\hat{\sigma}_{Q}$, the adaptive update laws are chosen as

$$
\begin{aligned}
& \dot{\hat{\sigma}}_{\alpha}=\gamma_{\alpha} \operatorname{Proj}\left(\hat{\sigma}_{\alpha},-\tilde{Q} \frac{\bar{q} \bar{S} \bar{c}}{I_{y}} c_{m \alpha} \alpha\right) \\
& \dot{\hat{\sigma}}_{\dot{\alpha}}=\gamma_{\dot{\alpha}} \operatorname{Proj}\left(\hat{\sigma}_{\dot{\alpha}},-\tilde{Q} \frac{\bar{q} \bar{S} \bar{c}}{I_{y}} \frac{\bar{c}}{2 V_{t}} c_{m \dot{\alpha}} \dot{\alpha}\right) \\
& \dot{\hat{\sigma}}_{Q}=\gamma_{Q} \operatorname{Proj}\left(\hat{\sigma}_{Q},-\tilde{Q} \frac{\bar{q} \bar{S} \bar{c}}{I_{y}} \frac{\bar{c}}{2 V_{t}} c_{m Q} Q\right),
\end{aligned}
$$

where $\operatorname{Proj}(\cdot)$ denotes the projection operator [12]. Then (69) becomes

$$
\dot{V}_{\text {pred }}=-L_{1} \tilde{\theta}^{2}-L_{2} \tilde{Q}^{2} \leq 0 \quad \forall \tilde{\theta}, \tilde{Q} \neq 0 .
$$




\subsubsection{Control Law}

We start by defining the error variables $z_{1}$ and $z_{2}$ as

$z_{1} \triangleq \theta-\theta_{d}$

$z_{2} \triangleq Q-\alpha_{1}$

where $\alpha_{1}$ is a stabilising function to be designed.

Consider the positive definite CLF

$V_{c t r l, 1}=\frac{1}{2} z_{1}^{2}$

whose derivative with respect to time along the $z_{1} \mathrm{dy}-$ namics becomes

$$
\begin{aligned}
\dot{V}_{c t r l, 1} & =z_{1} \dot{z_{1}} \\
& =z_{1}\left(\dot{\theta}-\dot{\theta}_{d}\right) \\
& =z_{1}\left(Q-Q_{d}\right) \\
& =z_{1}\left(z_{2}+\alpha_{1}-Q_{d}\right) .
\end{aligned}
$$

By substituting (75) into (77), the CLF becomes

$\dot{V}_{c t r l, 1}=z_{1} z_{2}+z_{1}\left(\alpha_{1}-Q_{d}\right)$.

The stabilising function can be chosen as

$\alpha_{1}=-K_{1} z_{1}+Q_{d}$,

where $K_{1}>0$, which gives

$\dot{V}_{c t r l, 1}=-K_{1} z_{1}^{2}+z_{1} z_{2}$.

The CLF is then extended to

$V_{c t r l, 2}=\frac{1}{2} z_{2}^{2}+V_{c t r l, 1}$,

such that it includes both $z_{1}$ and $z_{2}$. The derivative of the new CLF is

$$
\begin{aligned}
\dot{V}_{c t r l, 2}= & z_{2} \dot{z}_{2}+\dot{V}_{c t r l, 1} \\
= & z_{2}\left(\frac { \overline { q } \overline { S } \overline { c } } { I _ { y } } \left(c_{m 0}+\sigma_{\alpha} c_{m \alpha} \alpha+c_{m \delta e} \delta_{e}\right.\right. \\
& \left.\left.+\frac{\bar{c}}{2 V_{t}}\left(\sigma_{\dot{\alpha}} c_{m \dot{\alpha}} \dot{\alpha}+\sigma_{Q} c_{m Q} Q\right)\right)-\dot{\alpha}_{1}\right) \\
& -K_{1} z_{1}^{2}+z_{1} z_{2} \\
= & -K_{1} z_{1}^{2}+z_{2}\left(\frac { \overline { q } \overline { S } \overline { c } } { I _ { y } } \left(c_{m 0}+\sigma_{\alpha} c_{m \alpha} \alpha+c_{m \delta e} \delta_{e}\right.\right. \\
& \left.\left.+\frac{\bar{c}}{2 V_{t}}\left(\sigma_{\dot{\alpha}} c_{m \dot{\alpha}} \dot{\alpha}+\sigma_{Q} c_{m Q} Q\right)\right)-\dot{\alpha}_{1}+z_{1}\right) .
\end{aligned}
$$

The control law is chosen as

$$
\begin{aligned}
\delta_{e, c o m}= & \frac{-1}{c_{m \delta e}}\left(c_{m 0}+\hat{\sigma}_{\alpha} c_{m \alpha} \alpha+\frac{\bar{c}}{2 V_{t}}\left(\hat{\sigma}_{\dot{\alpha}} c_{m \dot{\alpha}} \dot{\alpha}\right.\right. \\
& \left.\left.+\hat{\sigma}_{Q} c_{m Q} Q\right)+\frac{I_{y}}{\bar{q} \bar{S} \bar{c}}\left(z_{1}-\dot{\alpha}_{1}+K_{2} z_{2}\right)\right),
\end{aligned}
$$

where $K_{2}>0$. It is assumed that uncertainties can be estimated perfectly through $\hat{\sigma}_{\alpha}, \hat{\sigma}_{\dot{\alpha}}$ and $\hat{\sigma}_{Q}$ using the state predictor as a cascade system. This leads to

$\dot{V}_{c t r l, 2}=-K_{1} z_{1}^{2}-K_{2} z_{2}^{2}<0 \quad \forall z_{1}, z_{2} \neq 0$.

The adaptation of the uncertainties may contain highfrequency signals. To avoid introducing such frequencies into the control input, a lowpass filter is introduced to the control signals such that

$\delta_{e, c}=C(s) \delta_{e, c o m}$,

where

$C(s)=\frac{k}{s+k}$,

is applied to the control signal and the gain $k>0$ is a design parameter.

\section{Simulation Results and Performance Evaluation}

This section first states the parameters of the aircraft model, followed by the initial states and control parameters used in the simulations. Subsequently, the metrics used to evaluate performance are defined. Finally, results associated with the different controllers are shown and discussed.

\subsection{Simulation Setup}

For simulation purposes, the controllers are implemented in Matlab. A Cessna 182 from [33] will be used to demonstrate the performance of the proposed methods. The parameter values for the aircraft are listed in Table 1 .

\begin{tabular}{cr||cc}
\multicolumn{2}{c}{ Table 1: Parameters for a Cessna } & $182[33]$ \\
\hline$m$ & $1202.02[\mathrm{~kg}]$ & $c_{m 0}$ & 0.04 \\
$g$ & $9.81\left[\mathrm{~m} / \mathrm{s}^{2}\right]$ & $c_{m \alpha}^{*}$ & -0.613 \\
$I_{y}$ & $56.72\left[\mathrm{~kg} \mathrm{~m}^{2}\right]$ & $c_{m \delta e}$ & -1.122 \\
$\bar{S}$ & $16.17\left[\mathrm{~m}^{2}\right]$ & $c_{m \dot{\alpha}}^{*}$ & -7.27 \\
$\bar{c}$ & $0.46[\mathrm{~m}]$ & $c_{m Q}^{*}$ & -12.4 \\
$\bar{q}$ & $2375.31\left[\mathrm{~N} / \mathrm{m}^{2}\right]$ & & \\
\hline
\end{tabular}

The elevator $\delta_{e}$ and ailerons $\delta_{a}$ have a limitation of [-22:18] and [-24:24] degrees in deflection angle, respectively. Here, the effectiveness matrix $\mathbf{W}(t)$ from $(22)$ is assumed to be known. The aerodynamic constants for the reconfigured ailerons are chosen to be $c_{L \delta a}=2 c_{L \delta e}$ and $c_{m \delta a}=\frac{1}{2} c_{m \delta e}$. 


\subsubsection{Initial States, Reference Signal, and Control Parameters}

The initial conditions of the aircraft are that it is flying straight and level, which means $\dot{Q}=\dot{\theta}=\dot{\alpha}=\dot{V}_{t}=0$ and $\theta=\alpha \approx 0$. The simulation starts at an altitude of $1524[\mathrm{~m}]$ and a true airspeed of $67[\mathrm{~m} / \mathrm{s}]$.

The uncertainties for the system are chosen as $\sigma_{\alpha}=4$, $\sigma_{\dot{\alpha}}=5$ and $\sigma_{Q}=7$. The initial values for the estimated uncertainties are $\hat{\sigma}_{\alpha}(0)=\hat{\sigma}_{\dot{\alpha}}(0)=\hat{\sigma}_{Q}(0)=1$. The elevator actuator fault is set to occur at $30[\mathrm{~s}]$.

It is desired for the aircraft to track a constant pitch angle $\theta_{\text {ref }}=10$ [deg] from $t=0$. To obtain a reference signal of $\theta_{d}$ that is in $\mathcal{C}^{2}$ and bounded, the constant pitch angle is put through a third-order lowpass filter with the structure

$\dot{\boldsymbol{\theta}}_{d}(t)=\mathbf{A} \boldsymbol{\theta}_{d}(t)+\mathbf{B} \theta_{r e f}$,

where $\boldsymbol{\theta}_{d}(t)=\left[\theta_{d}, Q_{d}, \dot{Q}_{d}\right]^{\top}$ and

$\mathbf{A}=\left[\begin{array}{ccc}0 & 1 & 0 \\ 0 & 0 & 1 \\ -\omega_{0}^{3}-(2 \zeta+1) \omega_{0}^{2}-(2 \zeta+1) \omega_{0}\end{array}\right] \quad \mathbf{B}=\left[\begin{array}{c}0 \\ 0 \\ \omega_{0}^{3}\end{array}\right]$

The initial condition of the reference signal is chosen to be $\boldsymbol{\theta}_{d}(0)=\left[0[\mathrm{deg}], 0[\mathrm{deg} / \mathrm{s}], 0\left[\mathrm{deg} / \mathrm{s}^{2}\right]\right]^{\top}$. Additionally, it is desirable to lower the true airspeed to the optimal climb speed of $50[\mathrm{~m} / \mathrm{s}]$, which is passed through a similar third-order lowpass filter to obtain a time-varying reference signal for the true airspeed.

The gain for designing the control law for the SMC is chosen to be $\varepsilon=0.005$ and $\beta_{0}=0.5$.

The L1-AB controller has a lowpass filter integrated in the control law to reject high frequency oscillations in the estimation of the uncertainties. Utilising this benefit, the control law for the L1-AB is designed with $K_{1}=21$ and $K_{2}=130$. The gains for the state predictor are chosen to be $L_{1}=L_{2}=300$. The adaptation gains for the estimation of the uncertainties are chosen to be $\gamma_{\sigma, \alpha}=\gamma_{\sigma, \dot{\alpha}}=\gamma_{\sigma, Q}=4000$. The gain for the lowpass filter is chosen to be $k=300$.

The baseline PID controller for the pitch motion has been implemented with the gains $K_{p, \theta}=1.5, \tau_{i}=1.5$, $\tau_{d}=0.15$ and $a=0.1$ obtained through tuning by assuming that the parameters $c_{m \alpha}, c_{m \dot{\alpha}}$ and $c_{m Q}$ are the true values. These control gains are chosen conservatively in order to cope with uncertainties in the aircraft model.

To control the airspeed during the simulation, a PI-controller has been implemented in a speed control loop. The gains $K_{P, V t}=20$ and $K_{I, V t}=0.5$ have been chosen for the proportional and integral gains, respectively.

\subsubsection{Performance Metrics}

To evaluate and compare the performance of the different control algorithms, it is advantageous to use performance metrics. These include the integral of the absolute error (IAE), integral of the square of the error (ISE) and integral of the absolute error multiplied by time (ITAE). Here, the error $e$ is defined as the pitch angle error

$e \triangleq \theta-\theta_{d}$.

The formula for the IAE is given as

$I A E=\int_{0}^{t}|e| d \tau$,

which simply describes the temporal evolution of the absolute value of the error without adding any weight to the error. The ISE is defined as

$I S E=\int_{0}^{t} e^{2} d \tau$

and penalises large errors more than smaller ones, indicating how good the particular algorithm is at eliminating large errors. The computation of ITAE is given as

$\operatorname{ITAE}=\int_{0}^{t} \tau|e| d \tau$,

which penalises errors which have been present for a long time more heavily than those at the beginning. ITAE will show if there is a steady error present in the system.

A final performance metric is also proposed, namely the integral of the absolute error multiplied by the energy consumption (IAEW), which can be computed by

$I A E W=\int_{0}^{t}|e| d \tau \int_{0}^{t} P d \tau$

where

$P=\left|Q \delta_{e, c}\right|$,

which represents the mechanical power and not the actual power consumption. Notice that commanded control input $\delta_{e, c}$, which is before the control allocation, is 
used for the calculation of $P$. The reason for considering IAEW is to get an indication of which control algorithm has the best combination of tracking performance and energy consumption.

\subsection{Results}

In the following sections, the simulation results of the different controllers are illustrated for both a fault-free and a faulty case. The performance metrics defined previously are used to evaluate the performance of the controllers.

\subsubsection{Fault-Free Case}

Fig. 4 displays the desired and actual trajectories of the state of the aircraft in the fault-free case, which shows that the methods are able to track the pitch angle and pitch rate. The PID controller is slower than the others, which is a trade-off by tuning it conservatively such that it can handle uncertainties. Additionally, it takes some time to achieve the desired true airspeed, which depends on how well the airspeed PI-controller has been tuned. Be advised that the length of the time axis is different in figures 4,5 and 6 , in order for the reader to easily see the properties of controllers.

The control signals are shown in Fig. 5, where the three controllers have the same control signal after $0.3 \mathrm{sec}-$ onds. Both the L1-AB and SMC have some high-frequency oscillations on the elevator in the start of the simulation. Since there is no fault present in the system there is no control signal distribution to the ailerons.

The tracking errors of the pitch angle and rate are displayed in Fig. 6, showing that the two nonlinear controllers track the pitch angle and rate fast, while the PID controller requires almost 20 seconds for the pitch angle error to go to zero. Additionally, the L1-AB has a small overshot and the PID is oscillating on the pitch angle, which makes them use more time to track the reference signal compared to the SMC. However, the L1$\mathrm{AB}$ and PID controllers have the advantage that they do not result in a stationary tracking error. The SMC is a robust controller without integral action, which is why it has a stationary tracking error.

\subsubsection{Faulty Case: Total Elevator Loss without Fault-dependent Control Allocation}

In the faulty case, the elevator goes to zero at $30 \mathrm{sec}$. The states in Fig. 7 show that the aircraft is no longer able to track the desired pitch angle and true airspeed
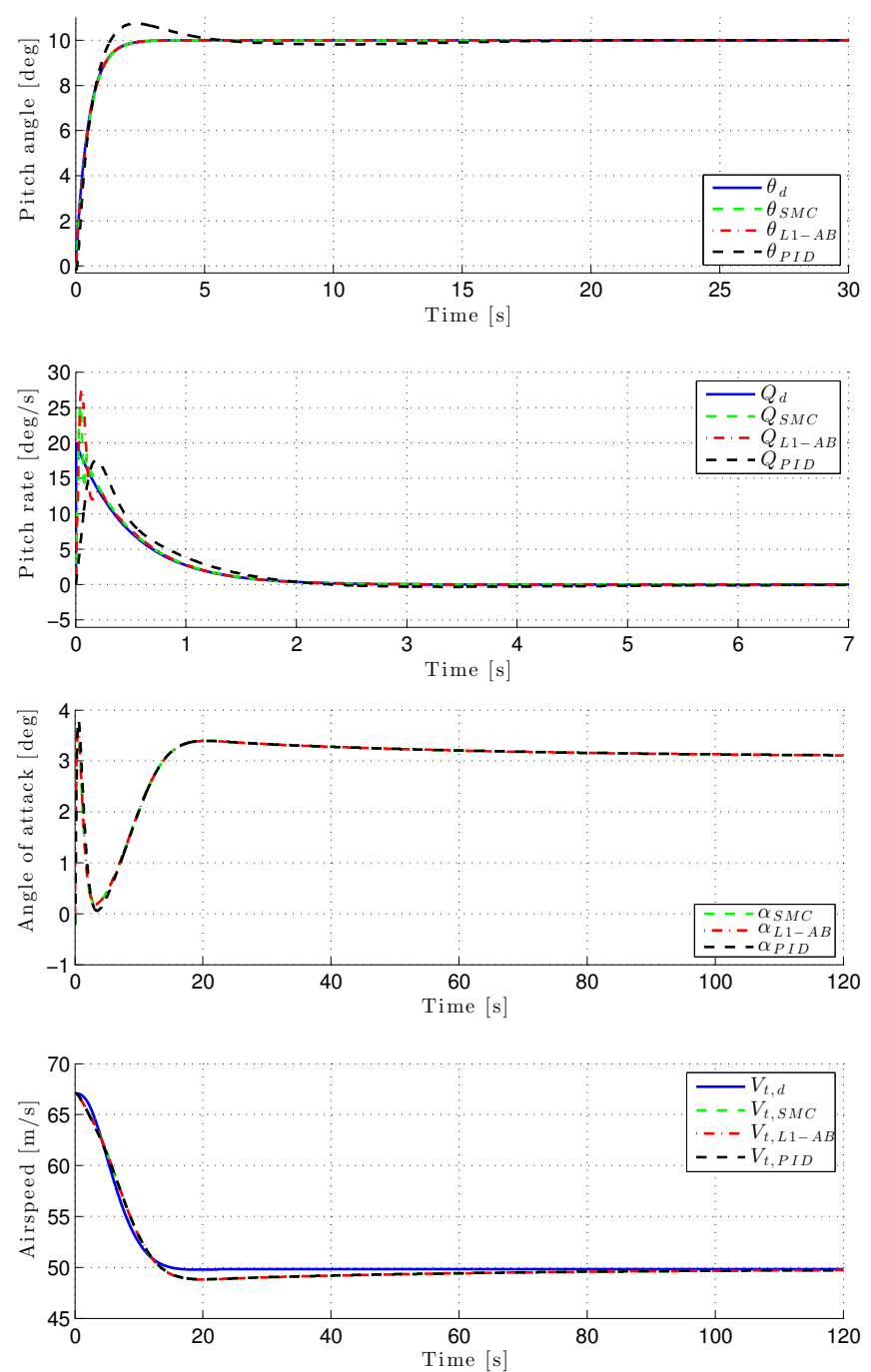

Fig. 4: States in the fault-free case

after the fault has occurred.

From Fig. 8 it can be concluded that this scenario has a high risk of going into an irreversible stall or spin. Since the elevator is stuck, there is no redundancy and the engine is already producing the maximum amount of power.

\subsubsection{Faulty Case: Total Elevator Loss with Fault-dependent Control Allocation}

As in Section 5.2.2, the elevator goes to zero and holds this position from 30 seconds. The results of loosing the elevator completely is displayed in Fig. 9-11. The only noticeable difference in the states compared to the fault-free case is the angle of attack, which has a smaller stationary value. 

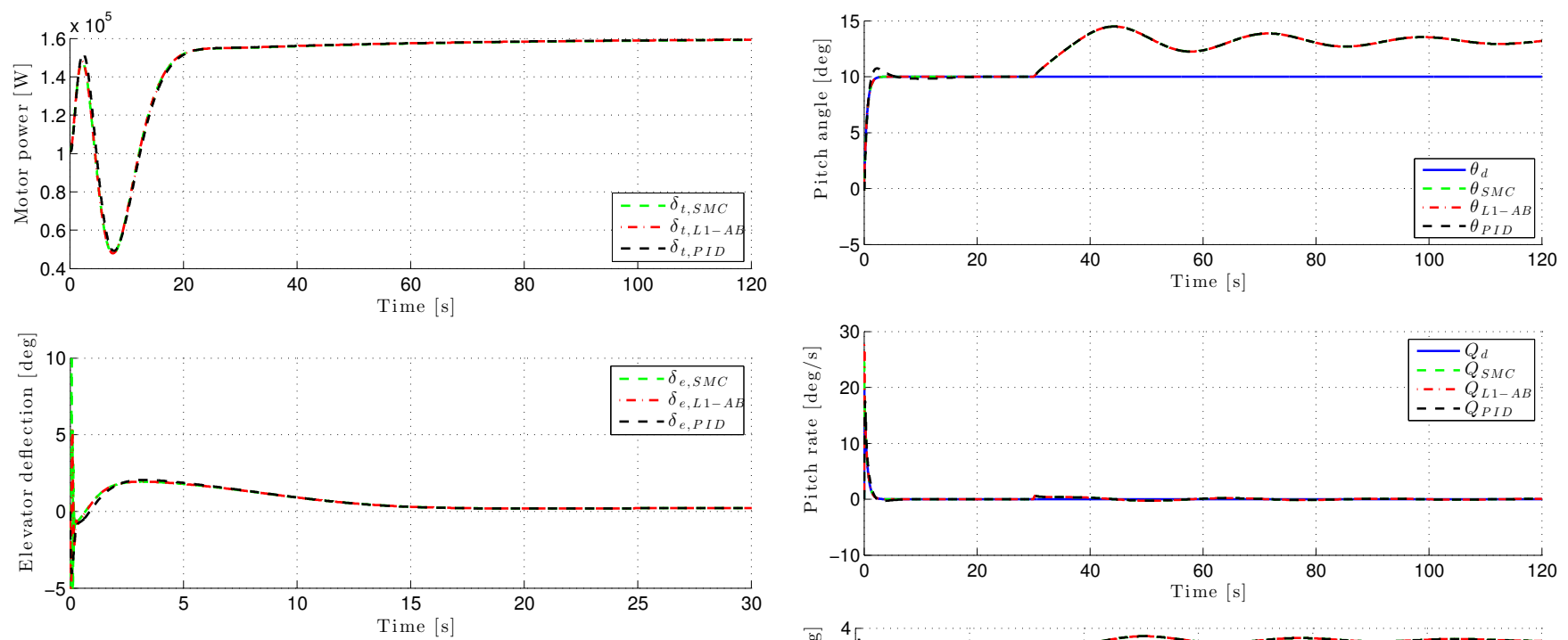

Fig. 5: Inputs in the fault-free case
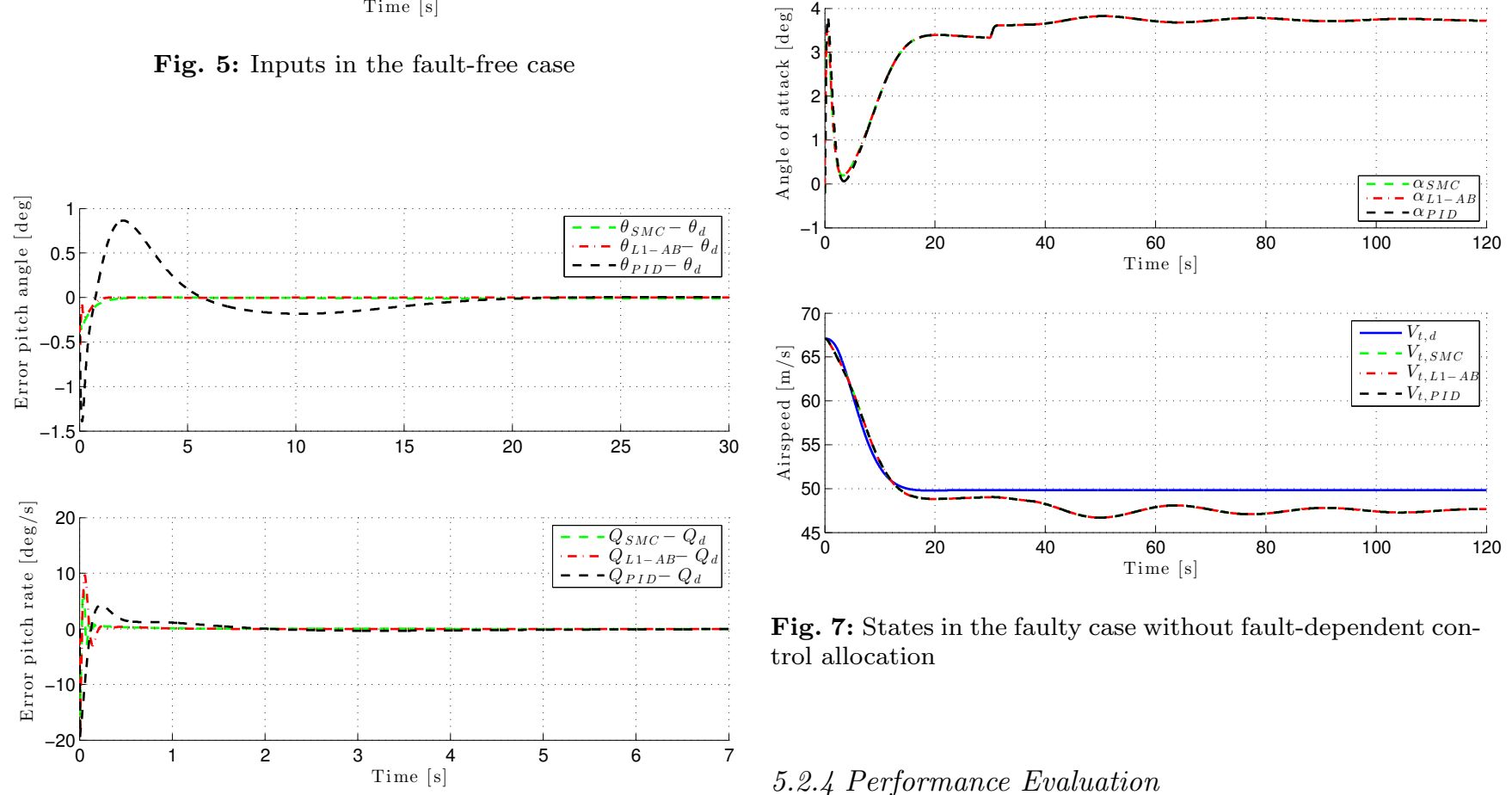

Fig. 7: States in the faulty case without fault-dependent control allocation

\subsubsection{Performance Evaluation}

Fig. 6: Errors in the fault-free case

By inspecting the inputs in Fig. 10, the change in the angle of attack $\alpha$ is caused by the amount of engine power. Additionally, Fig. 10 shows that when the effect of the elevator is lost, the ailerons become active since the control signal is reallocated to the ailerons.

Comparing the results with the fault-free case concerning the pitch angle and pitch rate tracking errors in figures 6 and 11 shows no degradation in the performance even when the elevator is faulty.

Figures 12 and 13 display the curves of IAE, ISE, ITAE and IAEW for the pitch angle tracking error. Fig. 12 clearly indicates that the L1-AB has the best performance both in terms of tracking and handling quicklyvarying signals. It is easier to see to from Fig. 12 that SMC has a stationary error since the IAE is constantly growing.

The ITAE shown in Fig. 13 clearly indicates that L1$\mathrm{AB}$ yields convergence of the pitch angle tracking error to zero. The plots of the ITAE illustrate that the SMC has a better performance than the PID until 75 seconds. In the evaluation between tracking performance versus energy consumption when uncertainties are affecting the system, the L1-AB controller performs best, 

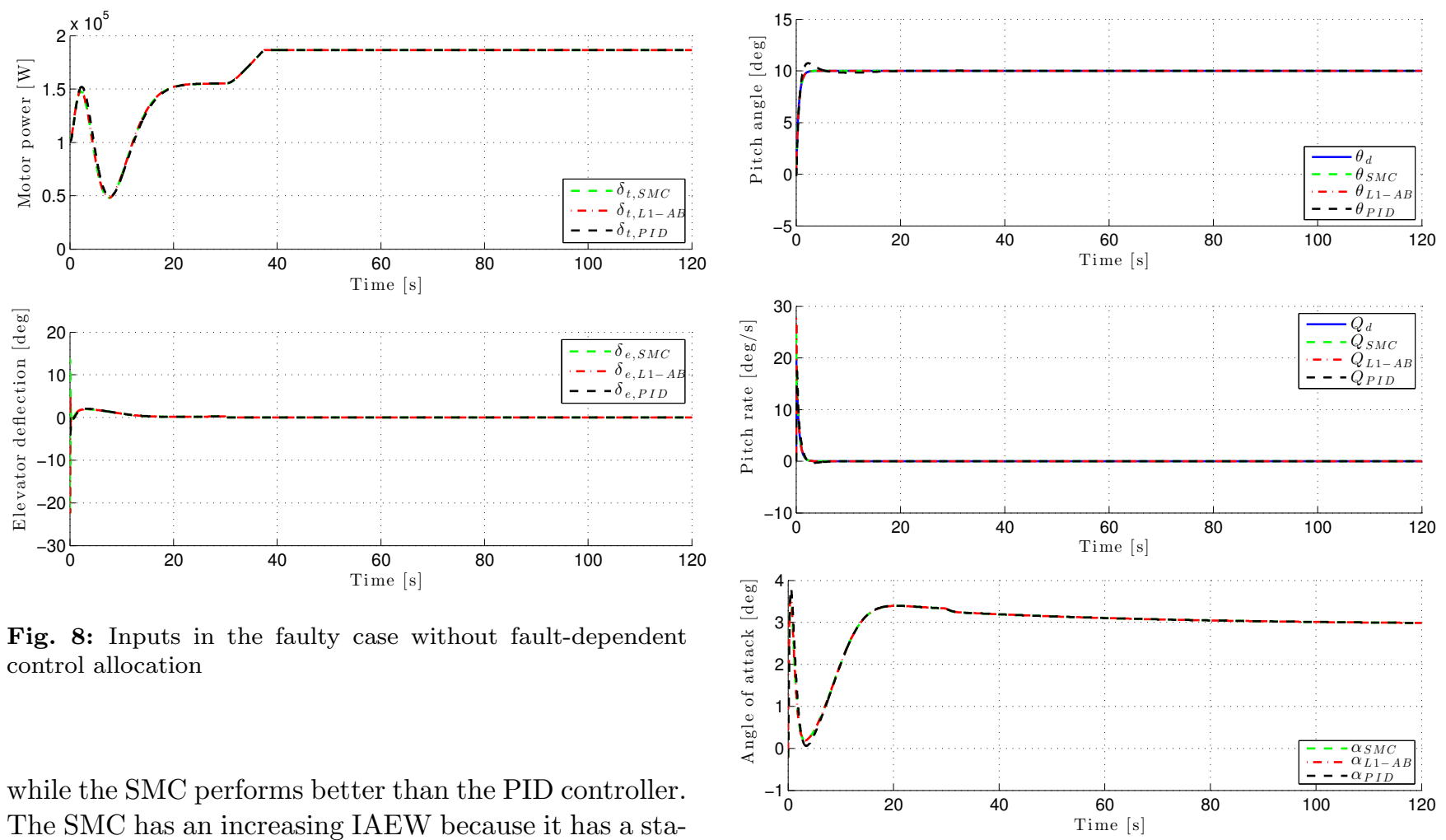

while the SMC performs better than the PID controller. The SMC has an increasing IAEW because it has a stationary error which is multiplied with a constant high energy use.

The simulations also show that the nonlinear control algorithms perform better than the PID controller. However, utilising the benefit which $\mathcal{L}_{1}$ adaptive backstepping control gives, we are able to choose higher adaptation rates and minimising the effects of highfrequency oscillations in the control signal and therefore get a better tracking performance than for SMC. The considered controllers have both advantages and disadvantages:

- The PID has a simple design, which makes it require less computational power than the nonlinear controllers. However, the PID is conservatively tuned since the aircraft model include uncertainties, which results in a slower tracking performance.

- The sliding mode controller is able overcome the model uncertainties, but it does not have integral action, which leads to a stationary tracking error.

- The $\mathcal{L}_{1}$ adaptive backstepping controller is able to overcome the model uncertainties and track the reference signal. However, in order to do so it requires a large amount of power for a small period of time and it has some high-frequency oscillations in the control signal.

In Table 2, the results of the following properties are listed on a scale of 1-5:

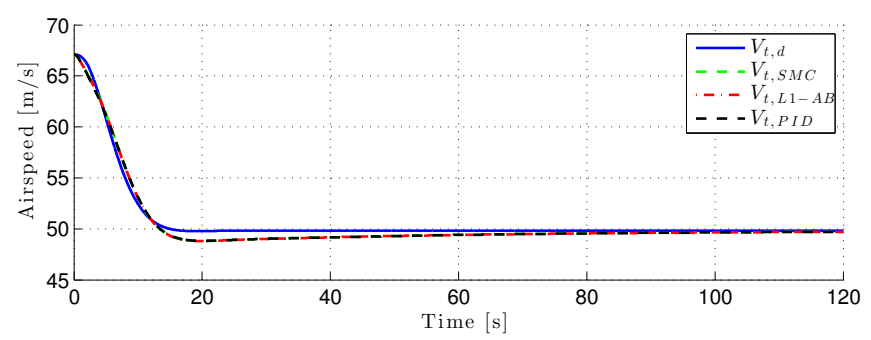

Fig. 9: States in the faulty case with fault-dependent control allocation

- Energy: The amount of energy the controller requires to perform the task.

- Convergence speed: How fast the controller converges to a set point.

- Robustness: How good the controller is in accommodating for uncertainties in the system.

- Control accuracy: How the controller's ability is to converge to and stay on target.

- Actuator stress: How much stress the controller puts on the actuator in order to perform the given task.

To validate that the advanced controllers are able to run in real time, the controllers were implemented as discrete-time algorithms running a sampling rate of $200 \mathrm{~Hz}$. Using Matlab/Simulink we are able to measure the execution time of the three controllers. The results are presented in Table 3, which shows that all 

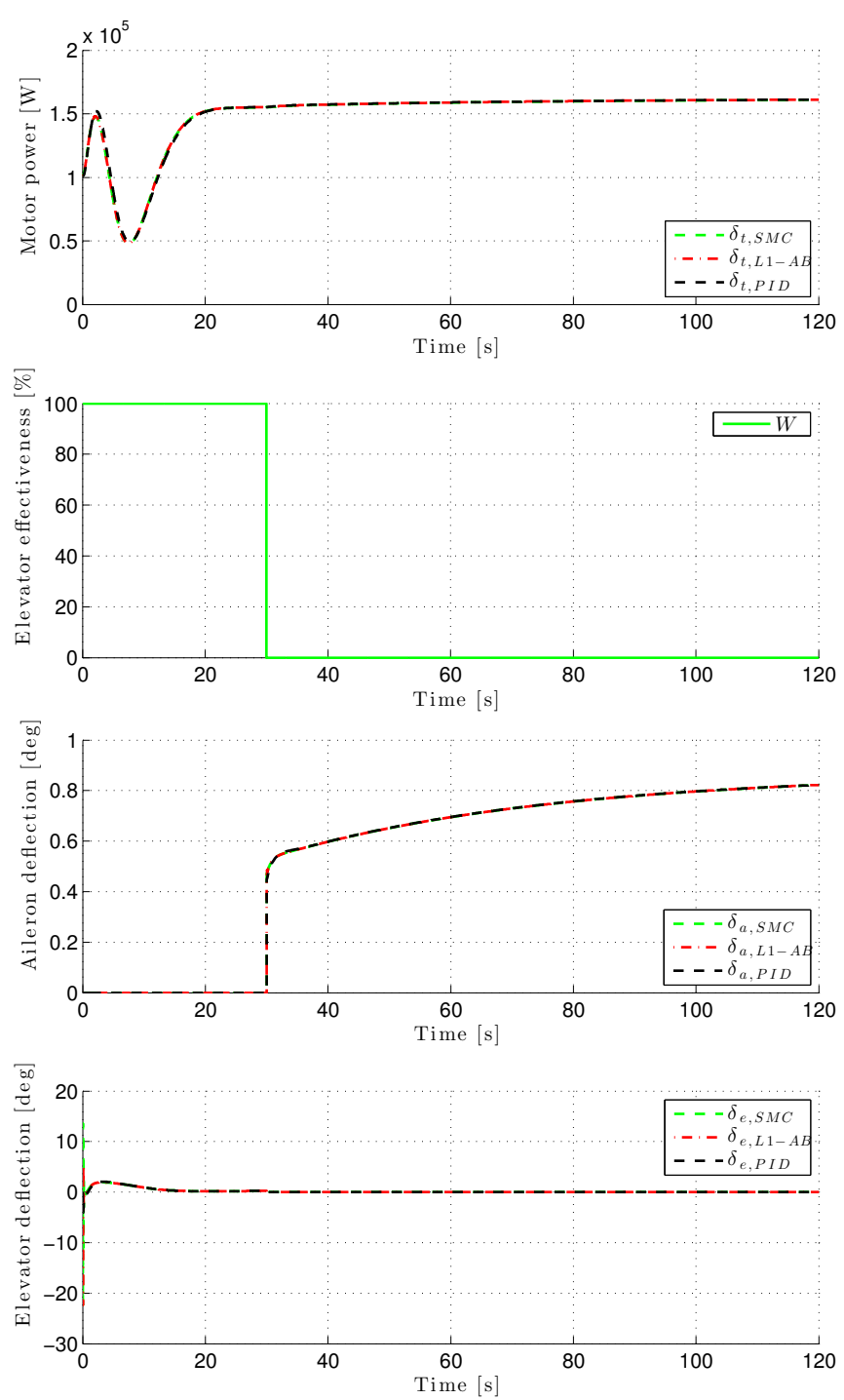

Fig. 10: Inputs in the faulty case with fault-dependent control allocation

Table 2: Strengths and weaknesses of the particular control algorithms in this evaluation. On a scale 1 (worst) to 5 (best)

\begin{tabular}{lccc} 
& PID & SMC & L1-AB \\
\hline Energy & 3 & 2 & 2 \\
Convergence speed & 3 & 4 & 5 \\
Robustness & 3 & 5 & 5 \\
Control accuracy & 3 & 2 & 4 \\
Actuator stress & 3 & 3 & 2 \\
\hline Total & 15 & 16 & 18
\end{tabular}

the controllers easily run in real time and the advanced controllers have an execution-time penalty that is below a factor of 2 compared with the PID. Hence, the $\mathcal{L}_{1}$ adaptive backstepping controller gives a better tracking
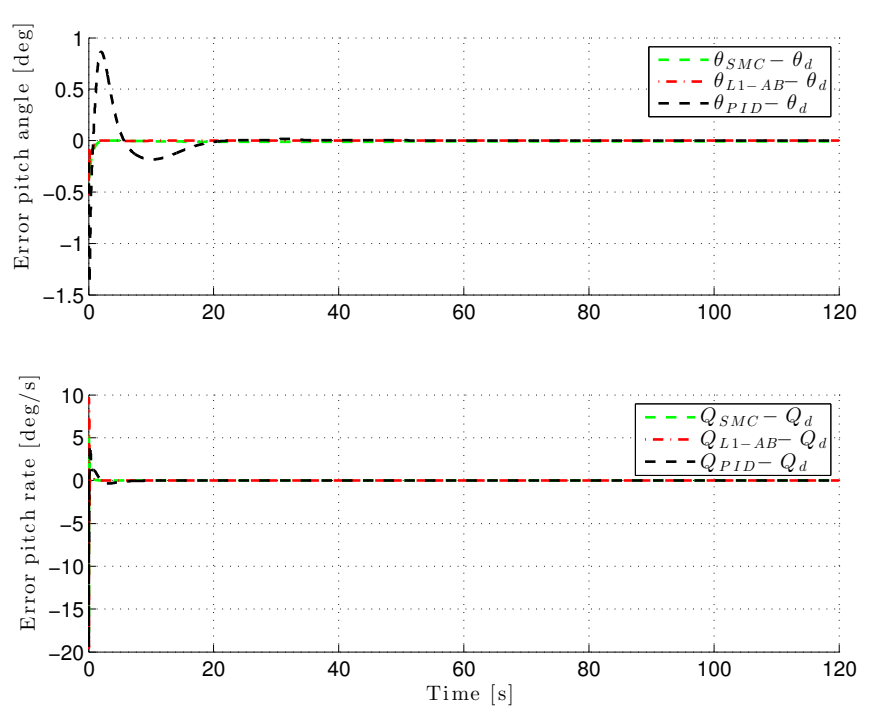

Fig. 11: Errors in the faulty case with fault-dependent control allocation
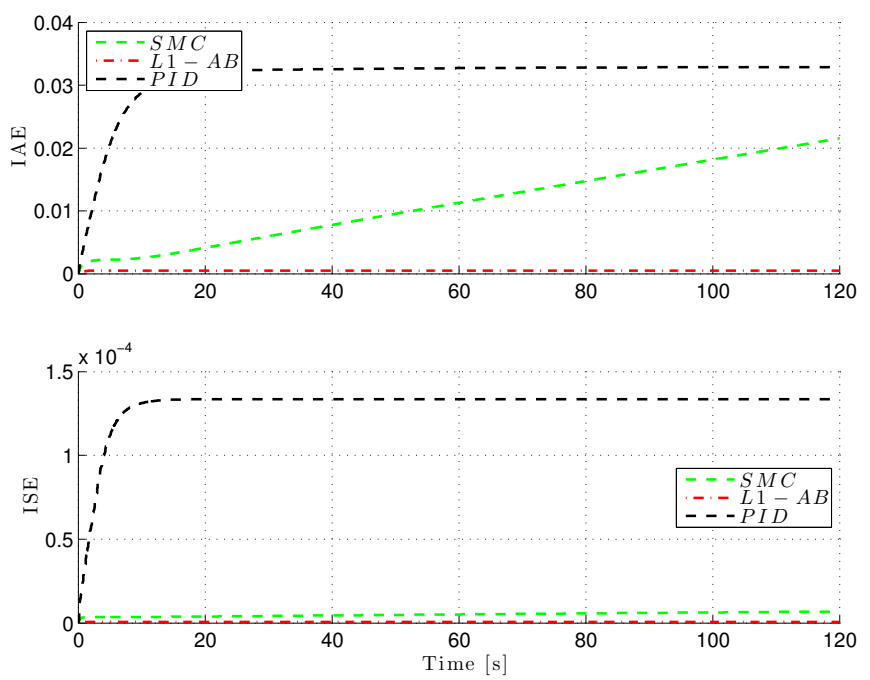

Fig. 12: The IAE and ISE of the pitch angle error in the fault-free scenario

performance in exchange for a slightly increased execution time.

Table 3: Execution time for the three controllers in a discrete simulation for modeling real-time performance

\begin{tabular}{lc} 
Controller & Execution Time \\
\hline PID & $90 \mu \mathrm{s}$ \\
SMC & $137 \mu \mathrm{s}$ \\
L1-AB & $160 \mu \mathrm{s}$ \\
\hline
\end{tabular}



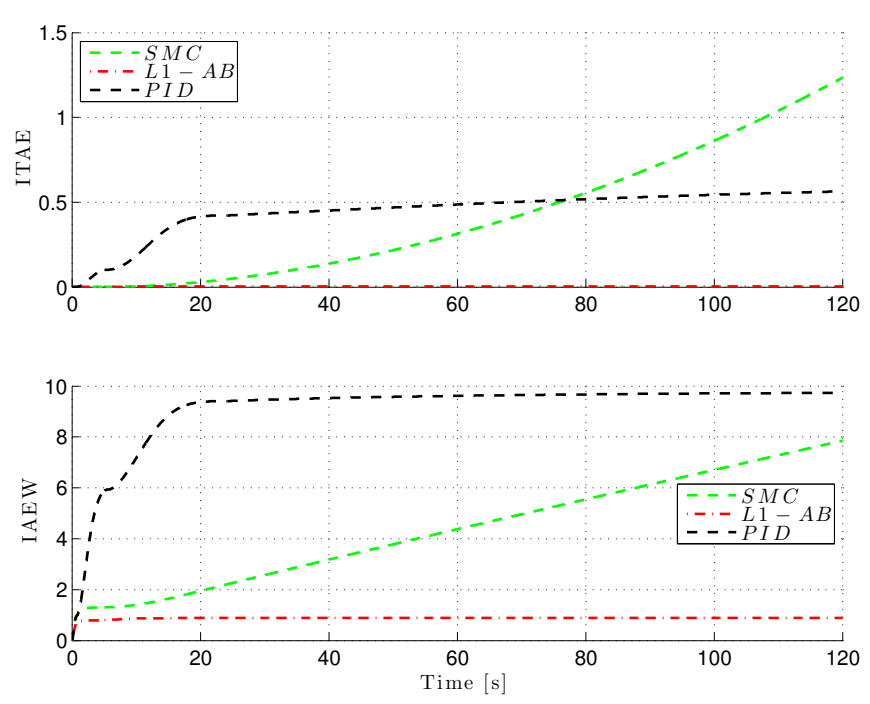

Fig. 13: The ITAE and IAEW of the pitch angle error in the fault-free scenario

\section{Conclusion}

This paper combined fault-dependent control allocation with three different control schemes to obtain fault tolerance in the longitudinal control of unmanned aerial vehicles. The paper has shown that fault-dependent control allocation is able to accommodate actuator faults that would otherwise be critical, and made a performance assessment for the different control algorithms: an $\mathcal{L}_{1}$ adaptive backstepping controller; a robust sliding mode controller; and a standard PID controller. This approach allowed the controllers to operate in nominal fault-free conditions using only the main actuator, which is the elevator. By adding fault-dependent control allocation which redistributes the control signal to redundant actuators, the system was shown to be fault tolerant against the total effective loss of the main actuator. A comparative analysis of the controllers was made in order to find out which had the best performance. Simulations were conducted on a high-fidelity model of a Cessna 182, showing that the considered controllers all have good tracking performance and the ability to compensate for model uncertainties. The results indicate that the fault-dependent control allocation scheme ensures excellent performance for both the nominal and faulty cases since the system is uniformly able to track a reference signal. The simulations also show that the nonlinear control algorithms perform better than the PID controller. From the performance metrics, it can be concluded that the $\mathcal{L}_{1}$ adaptive backstepping controller has the best overall performance. The advantages and disadvantages of the different con- trollers have been discussed. Also, by combining a controller with fault-dependent control allocation, it was shown that fault tolerance for the nonlinear longitudinal motion of an aircraft could be achieved.

Future work includes comparing the adaptive controller with an integral sliding mode controller, and proving stability and robustness of the closed-loop systems. Additionally, it is desirable to experimentally verify the results by implementing the methods on a UAV in a controlled environment.

\section{References}

1. C. Edwards, T. Lombaerts, and H. Smaili, Fault tolerant flight control: A benchmark challenge. Springer, 2010.

2. A. Zolghadri, "Advanced model-based FDIR techniques for aerospace systems: Today challenges and opportunities," Progress in Aerospace Sciences, vol. 53, pp. 18-29, 2012.

3. X. Qi, J. Qi, D. Theilliol, Y. Zhang, J. Han, D. Song, and C. Hua, "A review on fault diagnosis and fault tolerant control methods for single-rotor aerial vehicles," Journal of Intelligent and Robotic Systems, vol. 73, no. 1, pp. 535555, 2014.

4. P. Goupil, "Oscillatory failure case detection in the A380 electrical flight control system by analytical redundancy," Control Engineering Practice, vol. 18, no. 9, pp. 11101119, 2012.

5. E. Alcorta-Garcia, A. Zolghadri, and P. Goupil, "A nonlinear observer-based strategy for aircraft oscillatory failure detection: A380 case study," IEEE Transactions on Aerospace and Electronic Systems, vol. 47, no. 4, pp. 2792-2806, 2011.

6. S. Hansen and M. Blanke, "Control surface fault diagnosis with specified detection probability - Real event experiences," in Proceedings of the 2013 International Conference on Unmanned Aircraft System (ICUAS), Atlanta, USA, 2013.

7. D. Efimov, J. Cieslak, A. Zolghadri, and D. Henry, "Actuator fault detection in aircraft systems: Oscillatory failure case study," Annual Reviews in Control, vol. 37, pp. 180190, 2013.

8. M. Blanke and S. Hansen, "Towards self-tuning residual generators for UAVcontrol surface fault diagnosis," in Proceedings of the 2nd International Conference on Control and Fault-Tolerant Systems, Nice, France, 2013.

9. S. Hansen and M. Blanke, "Diagnosis of airspeed measurement faults for unmanned aerial vehicle," IEEE Transactions on Aerospace and Electronic Systems, vol. 50, no. 1, pp. 224-239, 2014.

10. Y. Shtessel, Sliding mode control and observation. Birkhauser, 2013.

11. C. Edwards and S. K. Spurgeon, Sliding mode control: Theory and applications. Taylor and Francis Ltd, 1998.

12. N. Hovakimyan and C. Cao, $\mathcal{L}_{1}$ adaptive control theory: Guaranteed robustness with fast adaptation. SIAM, 2010.

13. T. Espinoza, A. Dzul, R. Lozano, and P. Parada, "Backstepping - sliding mode controllers applied to a fixedwing UAV," Journal of Intelligent and Robotic Systems, vol. 73 , no. 1, pp. 67-79, 2014. 
14. C. Edwards and C. P. Tan, "Fault tolerant control using sliding mode observers," in Proceedings of the IEEE Conference on Decision and Control, Nassau, Bahamas, 2004.

15. H. Alwi and C. Edwards, "Fault tolerant control of a civil aircraft using a sliding mode based scheme," in Proceedings of the IEEE Conference on Decision and Control and European Control Conference, Seville, Spain, 2005.

16. H. Alwi and C. Edwards, "Fault tolerant control using sliding modes with on-line control allocation," Automatica, vol. 44, no. 7 , pp. $1859-1866,2008$.

17. H. Alwi, C. Edwards, O. Stroosma, and J. A. Mulder, "Simona flight simulator implementation of a fault tolerant sliding mode scheme with on-line control allocation," in Proceedings of the American Control Conference, Seattle, USA, 2008.

18. V. V. Patel, K. A. Wise, N. Hovakimyan, C. Cao, and E. Lavretsky, " $\mathcal{L}_{1}$ adaptive controller for tailless unstable aircraft in the presence of unknown actuator failures," in Proceedings of the AIAA Guidance, Navigation, and Control Conference, Hilton Head, South Carolina, USA, 2007.

19. C.-H. Lee, M.-J. Tahk, and B.-E. Jun, "Autopilot design for an agile missile using $\mathcal{L}_{1}$ adaptive backstepping control," in Proceedings of the 28th Congress of the International Council of the Aeronautical Sciences, Brisbane, Australia, 2012.

20. T. A. Johansen and T. I. Fossen, "Control allocation - A survey," Automatica, vol. 49, no. 5, pp. 1087-1103, 2013.

21. J. D. Boskovic and R. K. Mehra, "Control allocation in overactuated aircraft under position and rate limiting," in Proceedings of the American Control Conference, Anchorage, USA, 2002

22. K. Liu, J. Zhu, and B. Yu, "Longitudinal controller design for a fighter aircraft using $\mathcal{L}_{1}$ adaptive backstepping," in Proceedings of the World Congress on Intelligent Control and Automation, Taipei, Taiwan, 2011.

23. M. E. N. Sørensen and M. Breivik, "UAV fault-tolerant control by combined $\mathcal{L}_{1}$ adaptive backstepping and fault- dependent control allocation," in Proceedings of the IEEE Multi-Conference on Systems and Control, Sydney, Australia, 2015

24. C. Edwards, H. Alwi, and M. T. Hamayun, "Integral sliding mode fault tolerant control incorporating on-line control allocation," in Proceedings of the 11th International Workshop on Variable Structure Systems, Mexico City, Mexico, 2010.

25. M. T. Hamayun, H. Alwi, and C. Edwards, "An output integral sliding mode FTC scheme using control allocation," in Proceedings of the 50th IEEE Conference on Decision, Orlando, USA, 2011.

26. A. Bajodah, H. Tariq, K. Pakki, B. Chandra, R. Ahmed, and D.-W. Gu, "Fault tolerant control of aircraft actuating surfaces using generalized DI and integral SM control," Journal of Intelligent and Robotic Systems, vol. 69, no. 1, pp. 181-188, 2013.

27. G. J. J. Ducard, Fault-tolerant flight control and guidance systems: Practical methods for small unmanned aerial vehicles. Springer-Verlag, 2009.

28. B. L. Stevens and F. L. Lewis, Aircraft control and simulation. John Wiley and Sons, 2003.

29. D. Sanseverinati, Identification and Fault Diagnosis for Autonomous Aircraft. MSc thesis. Dept. of Electrical Eng., Technical University of Denmark, 2010.

30. M. E. N. Sørensen, Fault-Tolerant Control for Unmanned Aerial Vehicle. MSc thesis. Dept. of Electrical Eng., Technical University of Denmark, 2014.

31. M. E. N. Sørensen and M. Breivik, "Comparing nonlinear adaptive motion controllers for marine surface vessels," in Proceedings of the 10th IFAC Conference on Manoeuvring and Control of Marine Craft, Copenhagen, Denmark, 2015.

32. M. Blanke, M. Kinnaert, J. Lunze, and M. Staroswiecki, Diagnosis and fault-tolerant control. Springer, 2006.

33. J. Roskam, Airplane flight dynamics and automatic flight controls. DARcorporation, 2003.

34. H. K. Khalil, Nonlinear systems. Prentice Hall, 2002. 